\title{
Phase-Matched Third Harmonic Generation in a Plasma
}

\author{
J. M. Rax and N. J. Fisch
}

\begin{abstract}
Relativistic third harmonic generation in a plasma is investigated. The growth of a third harmonic wave is limited by the difference between the phase velocity of the pump and driven waves. This phase velocity mismatch results in a third harmonic amplitude saturation and oscillation. In order to overcome this saturation, we describe a phase-matching scheme based on a resonant density modulation. The limitations of this scheme are analyzed.
\end{abstract}

\section{INTRODUCTION}

$\mathbf{T}$ HIRD harmonic generation in a plasma has been recently proposed as a candidate for a coherent light source at very short wavelength [1]. The study of this process was triggered by recent advances in laser pulse compression [2]. One can now envision a laser plasma interaction in the relativistic regime, i.e., typically above $10^{18} \mathrm{~W} / \mathrm{cm}^{2}$.

At sugh high intensity, not only does the quiver velocity become relativistic, but the electron orbit becomes nonlinear and contains all the harmonics of the incident field [3].

In vacuum, this nonlinear orbit of an electron in an intense, plane-polarized, laser wave is the well-known figure " 8 " [4] depicted on Fig. 1. A cold plasma (with an electron temperature smaller than the electron rest mass, $511 \mathrm{keV}$ ), driven by a strong wave, can be viewed as an array of such microscopic nonlinear currents.

The coherence of these microscopic currents can thus ensure the growth or the decay of a coherent harmonic wave. Basically, three different emission processes must be considered. The first one, spontaneous harmonic emission, is indeed incoherent, and has been widely investigated [3]. Because of the lack of correlation between the various microscopic sources, the emitted power scales as the density, i.e., as the square of the plasma frequency $\omega_{p}^{2}$. If these microscopic currents are properly correlated, we obtain collective harmonic emission (which will be considered in this work), and is what we call harmonic generation. In this process, the emitted wave is too weak to modify the electron dynamics driven by the incident wave. The stimulated harmonic emission, when the induced wave feeds back on the electron dynamics, which might boost the linear growth to an exponential one, is not considered in this paper.

In a cold plasma, the relative phasing of the currents is ensured by the pump wave. This emission process is collective,

Manuscript received May 10, 1992; revised September 14, 1992. This work was supported by the U. S. Department of Energy under Contract DE-AC0276-CHO3073

The authors are with the Plasma Physics Laboratory, Princeton University, Princeton, NJ 08540.

IEEE Log Number 9206344.

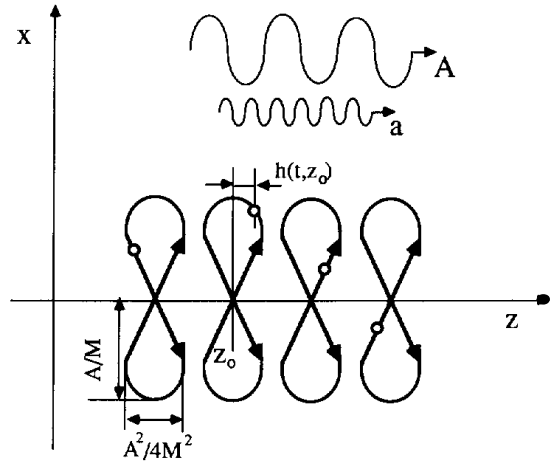

Fig. 1. A cold plasma, driven by an intense wave $A$, can be viewed as an array of microscopic currents including both linear and nonlinear responses to the applied field. The main difficulty in calculating the amplitude of the various processes is to sum accurately the individual nonlinear responses.

but not stimulated, so that a linear growth of the harmonic wave is expected. In fact, it has been shown previously [5] that a phase-locked linear regime does not occur for a long time, and, since the phase velocity of the driven and pump waves are different, a very rapid unlocking takes place, resulting in an amplitude oscillation. The purpose of this paper is to study in greater detail both this process and the means to overcome the associated saturation.

This paper is organized as follows. In Section II, we calculate the nonlinear currents induced by an intense laser wave in a cold plasma. To carry out this program, we use a Lagrangian description of the plasma response [6], and a density expansion scheme [7]. This is a powerful approach, particularly because, in the relativistic regime, the plasma frequency is to be replaced by an effective plasma frequency due to the electron effective mass. In Section III we study the dynamics of the harmonic wave driven by the nonlinear currents. We show that this dynamics can be reduced to the study of the amplitude and phase evolution of the generated wave. In Section IV, to overcome the phase-velocity mismatch saturation, the use and limitations of a resonant density modulation are analyzed. Section $\mathrm{V}$ gives our conclusions.

In the following calculations, except in the discussion, we will use $e=m=c=\omega=1$, where $e$ and $m$ are the electron charges, and mass $c$ is the velocity of light, and $\omega$ the laser pump frequency.

\section{NONLINEAR CURRENTS}

Two regimes of relativistic laser plasma interaction have been identified, the so-called short pulse, and long pulse 
regime [1]. In the former, the plasma period $\omega_{p}^{-1}$ is longer than the pulse duration $\delta \omega^{-1}$, and in the latter regime this time ordering is reversed. In the short pulse regime, the pulse-particle interaction is dominated by the relativistic ponderomotive force [7], which acts as a ballistic perturbation on the electron population. The main effect of this perturbation is to create an electrostatic wake behind the pulse. On the other hand, in the long pulse regime, the picture is completely different; after a transient response at the front edge of the pulse, a nonlinear oscillation, driven by the wave, and modulated by the plasma collective effects, is set up in the bulk of the pulse. Thus, provided that the vector potential envelop of the laser pulse, $A(z, t)$, is slowly varying $(\partial A / \partial z<\omega A / c, \partial A / \partial t<\omega A)$, when an electron enters the pulse, it behaves essentially as in an infinite wave. Consequently, in this section, we will neglect the influence of the pump-envelop dynamics, i.e., we will neglect the group velocity mismatch. This latter effect is an edge effect. It takes place on a long time scale compared to the phase velocity mismatch [5]. It is therefore the phasevelocity mismatch that is responsible for the inefficiency of third harmonic generation in an homogeneous plasma.

A laser pulse is said to be an ultrahigh-intensity pulse if $e A / m c \geq 1$; above this $10^{18} \mathrm{~W} / \mathrm{cm}^{2}$ threshold, the electron quiver velocity is fully relativistic. In this relativistic regime, as we show later, the calculation of the dispersion relation involves the plasma frequency, but the electron mass $m$ is to be replaced by the electron effective mass $M$, so that $\omega_{p}^{2} /\left(\omega^{2} M\right) \approx \omega_{p}^{2} /\left(\omega^{2} e A / m c\right)$ is a small parameter, even near the linear cutoff, when $A>1$. Thus, the ratio of the effective plasma frequency to the pump frequency provides a small parameter that may be employed to carry out expansion schemes. Moreover, the physical interpretation of such a density expansion scheme is clear. Consider an intense, linearly polarized, laser wave propagating along the $z$ axis, with

$$
\boldsymbol{A}(z, t)=A \cos [t-z+\theta(t)] \boldsymbol{e}_{x}
$$

where $\theta(t)$ is a slowly varying phase, to be determined, describing the nonlinear dispersion of the phase velocity. This field drives the electron motion through the Lorentz equation. Each electron is described by its unperturbed position $z_{o}$, and it follows a Lagrangian orbit, $x\left(z_{o}, t\right)$, and

$$
h\left(z_{o}, t\right)=z(t)-z_{o}
$$

about its rest position (Fig. 1). The momentum, $\boldsymbol{p}=d x / d \tau \mathbf{e}_{x}+$ $d h / d \tau \boldsymbol{e}_{z}$, and energy $\gamma^{2}=1+(d x / d \tau)^{2}+(d h / d \tau)^{2}(\tau$ is the proper time) of the electrons are solution of the equations.

$$
\left\{\begin{array}{l}
\frac{d \boldsymbol{p}}{d t}=\frac{\partial \boldsymbol{A}}{\partial t}-\frac{\boldsymbol{p}}{\gamma} \times \frac{\partial}{\partial \boldsymbol{r}} \times \boldsymbol{A}-\omega_{p}^{2} h \boldsymbol{e}_{z} \\
\frac{d \gamma}{d t}=\frac{\boldsymbol{p}}{\gamma} \cdot \frac{\partial \boldsymbol{A}}{\partial t}-\omega_{p}^{2} h \frac{d h}{d t} .
\end{array}\right.
$$

The last term on the right-hand side of these equations is a restoring force, which arises from the application of the Gauss theorem to the perturbed electron density [6]. This electrostatic force describes the collective plasma response so that, in this Lagrangian representation, the use of a scalar potential is avoided. The solution of the Lorentz equations (3) allows us to calculate the Eulerian current due to the response of one electron

$\boldsymbol{J}(z, t)=\frac{d x}{d t} \delta\left[z-z_{o}-h\left(t, z_{o}\right)\right] \boldsymbol{e}_{x}=\boldsymbol{A}(z, t) \frac{\delta\left[z-z_{o}-h\left(t, z_{o}\right)\right]}{\gamma\left(z_{o}, t\right)}$

where $\delta$ is the Dirac function, and we have used the conservation of the transverse canonical momentum, $\gamma d x / d t=A$.

We have to perform the sum of all these nonlinear currents in order to calculate the source terms in the Maxwell's equations. In doing so, we set up a closed, coupled, description of the wave and particle dynamics. Using the Lorentz gauge, we obtain

$$
\frac{\partial^{2} \boldsymbol{A}}{\partial z^{2}}-\frac{\partial^{2} \boldsymbol{A}}{\partial t^{2}}=\omega_{p}^{2} \boldsymbol{A}(z, t) \int d z_{o} \frac{\delta\left[z-z_{o}-h\left(t, z_{o}\right)\right]}{\gamma\left(z_{o}, t\right)} .
$$

To solve the coupled Maxwell-Lorentz system (3) and (5), let us first consider the lowest order in $\omega_{p}^{2}$. When all collective plasma effects are neglected, the electrons perform the wellknown figure "8" motion [4] depicted in Fig. 1,

$$
\left\{\begin{array}{l}
x=\frac{A}{M} \sin [M \tau+\theta] \\
h=\frac{A^{2}}{8 M^{2}} \sin [2 M \tau+2 \theta]
\end{array}\right.
$$

where we have introduced the effective mass of the electron in the wave $A$,

$$
M=\sqrt{1+A^{2} / 2} .
$$

The proper time $\tau$ is a nonlinear function of the time, which contains all the harmonics of the incident pump wave. This nonlinear relation between the time and the proper time is the key issue in the calculation of the electron nonlinear response, $t=z_{o} / V+\int^{\tau} \gamma(u) d u, V$ is the slope of the space-time translation through which we obtain all the orbits from a particular one as depicted in Fig. $2 . V$ is equal to one to lowest order, and to the phase velocity inside the pulse. On the basis of (6), we can express $\tau$ in terms of $J_{n}$, the ordinary Bessel functions, as

$\tau\left(t, z_{0}\right)=\frac{t-z_{o} / V}{M}+\sum_{n=1}^{n=\infty} \frac{J_{n}\left[-n A^{2} / 4 M^{2}\right]}{M n} \sin \left[2 n\left(t-z_{o}\right)+2 n \theta\right]$.

After some algebra, we can rewrite $\int d z_{o} \delta\left[z-z_{o}-\right.$ $\left.h\left(t, z_{o}\right)\right] \gamma^{-1}\left(z_{o}, t\right)=(\gamma-p)^{-1}$ to lowest order, where $p$ is the longitudinal momentum, $p=d h / d \tau$. From (6) we obtain $\gamma-p=M$. Thus, despite the fact that the microscopic Lagrangian currents contain the various harmonics of $\omega$, the Eulerian current contains only the fundamental, so that the Maxwell equation (5) becomes

$$
\frac{\partial^{2} \boldsymbol{A}}{\partial z^{2}}-\frac{\partial^{2} \boldsymbol{A}}{\partial t^{2}}=\frac{\omega_{p}^{2}}{M} \boldsymbol{A}
$$

This absence of harmonic generation, to lowest order in $\omega_{p}^{2}$, is not surprising. Here we are looking for an $\omega_{p}^{4}$ collective response and, to get this response, we have to calculate the nonlinear orbit to first order in $\omega_{p}^{2}$, for the Eulerian currents to be second order in $\omega_{p}^{2}$.

Nevertheless, despite the absence of harmonic generation to this order, the relativistic nonlinearity manifests itself through 


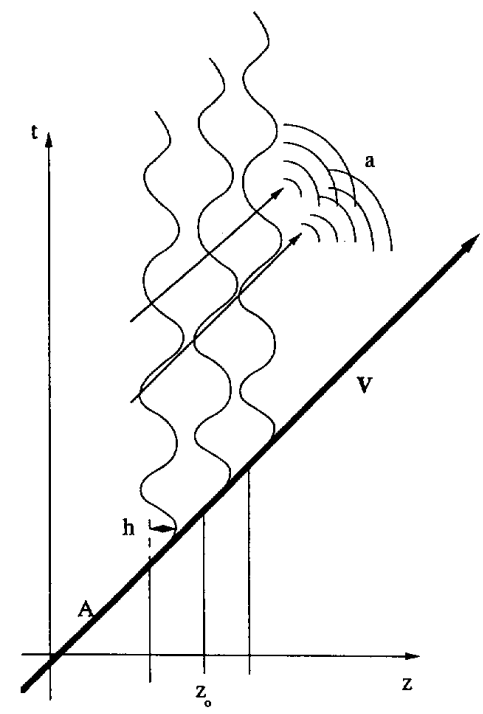

Fig. 2. Lagrangian picture of the electron dynamics in an intense plane wave The relative phasing of the electrons is given by the slope of the wave front $V$.

the slowly varying phase evolution which can be obtained by plugging (1) into (9) to get

$$
\theta(t)=\frac{\omega_{p}^{2}}{2 M} t
$$

Thus, as a result of the cancellation between the relativistic velocity anharmonicity and the relativistic density oscillations, harmonic generation occurs only at the order $\omega_{p}^{4}$, i.e., at the order $\omega_{p}^{2}$ for the orbits [1]. In this order, the phase velocity is $1+\omega_{p}^{2} / 2 M$, and we have to solve (3), to first order in $\omega_{p}^{2}$. Equation (3) gives

$$
\left\{\begin{array}{l}
\frac{d p}{d \tau}=-\frac{A^{2}}{2} \sin [2(t-z)+2 \theta]-\omega_{p}^{2} \gamma h \\
\frac{d \gamma}{d \tau}=-\frac{A^{2}}{2} \sin [2(t-z)+2 \theta]\left[1+\frac{d \theta}{d t}\right]-\omega_{p}^{2} p h
\end{array}\right.
$$

and we seek a solution of the form: $h \approx\left(A^{2} / 8 Q^{2}\right) \sin [2 Q \tau+$ $2 \theta]+O\left[\omega_{p}^{2}\right]$, and $t \approx Q \tau+\left(A^{2} / 8 Q^{2}\right) \sin [2 Q \tau+2 \theta]+O\left[\omega_{p}^{2}\right]$. The plasma effects not only add up higher order harmonic terms, $O\left[\omega_{p}^{2}\right]$, to the solution (6), but also shift the nonlinear frequency $M$ to give a new effective mass, $Q=M+$ $O\left[\omega_{p}^{2}\right]$. This nonlinear frequency shift is the standard way to avoid secular terms in the perturbative solution of nonlinear oscillation equations. After some algebra, we obtain

$\int d z_{o} \frac{\delta\left[z-z_{o}-h\left(t, z_{o}\right)\right]}{\gamma\left(z_{o}, t\right)}=\left[Q+\omega_{p}^{2} \frac{3 A^{2}}{16 Q^{2}} \cos (2 Q \tau+2 \theta)\right]_{(12)}^{-1}$

where $Q \tau=t-z+O\left[\omega_{p}^{2}\right]$. In fact, we do not need to calculate the exact value of $Q$ [5]. To study harmonic generation, the important point is that there exists a steady-state oscillation solution of (11), at the end, as we shall see, we will keep the lowest relevant order in $\omega_{p}^{2}$, but the $O\left[\omega_{p}^{2}\right]$ correction to $M$ will not come into play.
Equation (12) allows us to write down the Eulerian nonlinear current up to the order $\omega_{p}^{4}$,

$$
-\mu_{\mathrm{o}} \boldsymbol{J}(t, z)=\boldsymbol{A}(t, z)\left[\frac{\omega_{p}^{2}}{Q}-\frac{\omega_{p}^{4} 3 A^{2}}{16 Q^{4}} \cos [2(t-z)+2 \theta]\right] .
$$

The nonlinear current clearly contains the third harmonic of the pump field. However, this current is phase locked with the phase velocity of the pump wave, which is expected to be different from the phase velocity of the driven third harmonic wave. Thus, we have to plug (13) into the Maxwell equation in order to find the efficiency of harmonic generation.

\section{THIRD HARMONIC GenERATION}

Since the current response contains a third harmonic component, rather than (1), we have to seek a solution of Maxwell's equations in the form of a sum of the pump field $A$, plus a third harmonic field $a$. To study the dynamics of this third harmonic, we consider the slowly varying wave

$\boldsymbol{A}(z, t)=A \cos [t-z+\theta(t)] \boldsymbol{e}_{x}+a(t) \cos [3(t-z)+\phi(t)] \boldsymbol{e}_{x}$.

The amplitude $a$ and phase $\phi$ are evolving on the slow time scale of the problem. Because of the conservation of the transverse canonical momentum, (13) is still valid, with $\boldsymbol{A}$ given by (14). We separate the fundamental from the harmonics to obtain

$$
\begin{aligned}
& {\left[\frac{\partial^{2}}{\partial z^{2}}-\frac{\partial^{2}}{\partial t^{2}}\right] a(t) \cos [3(t-z)+\phi(t)]} \\
& =\frac{\omega_{p}^{2}}{M} a(t) \cos [3(t-z)+\phi(t)]-\frac{\omega_{p}^{4} 3 A^{3}}{32 M^{4}} \cos [3(t-z)+3 \theta(t)] .
\end{aligned}
$$

The second term, on the right-hand side, induces the amplitude dynamics, while the first term is reactive and describes refraction.

Then we use the slowly varying amplitude and phase assumption and average out the fast time scale. After the change of phase variable, $\varphi(t)=\phi(t)-3 \theta(t)$, we find to the lowest relevant order in $\omega_{p}^{2}$

$$
\left\{\begin{array}{l}
\frac{d a}{d t}=-\omega_{p}^{4} \frac{A^{3}}{64 M^{4}} \sin (\varphi) \\
\frac{d \varphi}{d t}=-\frac{4 \omega_{p}^{2}}{3 M}-\omega_{p}^{4} \frac{A^{3}}{64 M^{4}} \frac{\cos (\varphi)}{a} .
\end{array}\right.
$$

The phase portrait of this dynamical system is depicted in Fig. 3, where we show the level curves of the invariant

$$
C=a^{2}+\omega_{p}^{2} \frac{3 A^{3}}{128 M^{3}} a \cos (\varphi)
$$

If it starts from background noise, the wave describes the separatrix orbit on Fig. 3, $C=0$, so that the amplitude $a$ oscillates between

$$
-\omega_{p}^{2} \frac{3 A^{3}}{128 M^{3}}<a<\omega_{p}^{2} \frac{3 A^{3}}{128 M^{3}} .
$$




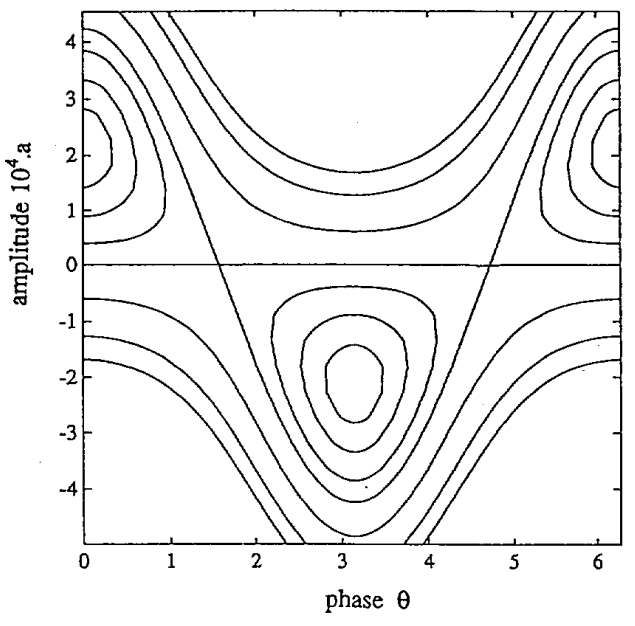

Fig. 3. Phase portrait of $(16), e A / m c=1,\left(\omega_{p} / \omega\right)^{2}=0.1$.

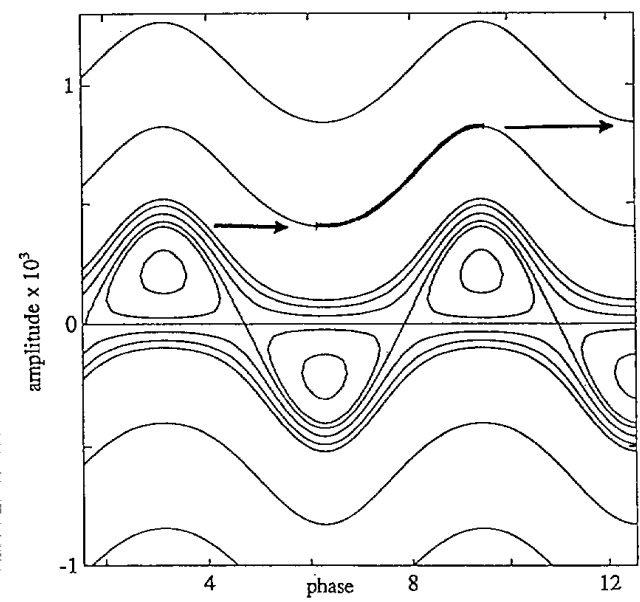

Fig. 4. If it starts from background noise, the third harmonic wave describes the separatrix orbit. To detrap this orbit, one can kick the wave repeatedly, i.e., resonantly, along the bold arrows.

The ratio of the third harmonic power $P_{3}$ to the pump power $P_{1}$ remains bounded at a low level [5] namely,

$$
\frac{P_{3}}{P_{1}} \approx\left(\frac{9}{128}\right)^{2}\left(\frac{\omega_{p}}{\omega}\right)^{4} \frac{A^{4}}{M^{6}} .
$$

To overcome this saturation, and to increase the efficiency of harmonic generation, we have to find a means to detrap this oscillating orbit.

\section{Phase Matching}

Detrapping can be achieved in a resonant way. Consider Fig. 4, when the amplitude $a$ reaches the maximum of the separatrix orbit; suppose we were able to kick the wave to the minimum of a different orbit in the direction of the bold arrows. Then, the wave evolves along the bold orbit. Again, at the maximum, we could transfer it to another orbit, and so on.

Such a scheme, which alternates passive dephasing (arrows) with active generation can be implemented with high and low density plasma layers. Rather than a square wave density modulation, it seems more practical to consider a sine wave density modulation such as an ion acoustic wave

$$
\frac{\delta n}{n}=\varepsilon \sin \left(\Omega_{i} t-K_{i} z\right)
$$

where $n$ is the density, $\varepsilon<1$, and the dispersion relation is $\Omega_{i}=K_{i} \sqrt{T / m_{i}}$ ( $T$ is the electron temperature, and $m_{i}$ is the ion mass). Typically, the pulse length is smaller than the ion acoustic wavelength, so that the modulation is basically static on the time scale of the laser pulse dynamics. Thus, we introduce the modulation frequency seen by the pulse, $\Omega=\Omega_{i} v_{g} / \sqrt{T / m_{i}}$, where $v_{g}=c\left(1+O\left[\left(\omega_{p} / \omega\right)^{2}\right]\right)$ is the group velocity of the pulse. To the lowest relevant order in $\omega_{p} / \omega$, we can take $\Omega \approx \Omega_{i} / \sqrt{T / m_{i}}$. Equation (16) then becomes

$$
\left\{\begin{array}{l}
\frac{d a}{d t}=\frac{\omega_{p}^{4} A^{3}}{64 M^{4}}[1+2 \varepsilon \sin (\Omega t)] \sin (\varphi) \\
\frac{d \varphi}{d t}=-\frac{4 \omega_{p}^{2}}{3 M^{4}}[1+\varepsilon \sin (\Omega t)] \\
-\frac{\omega_{p}^{4} A^{3}}{64 M^{4}}[1+2 \varepsilon \sin (\Omega t)] \frac{\cos (\varphi)}{a} .
\end{array}\right.
$$

When the amplitude $a$ becomes large, the second term on the right-hand side of the second equation can be neglected compared to the first one, and the phase oscillates with time. Then we plug this phase oscillation into the amplitude equation, and use the Bessel expansion of the sine of an oscillation, so that we can write to lowest relevant $\varepsilon$ order

$$
\frac{d a}{d t}=-\frac{\omega_{p}^{4} A^{3}}{64 M^{4}} \sum_{N} J_{N}\left(-4 \varepsilon \omega_{p}^{2} / 3 M \Omega\right) \sin \left[\frac{4 \omega_{p}^{2}}{3 M} t+N \Omega t+N \frac{\pi}{2}\right] .
$$

Clearly, for some particular choices of the parameters, $N, \Omega$, and $\omega_{p}$, one can induce a secular linear response.

To do so we take a particular odd integer $N$ and consider the resonance condition, $N \Omega+\left(4 \omega_{p}^{2} / 3 M\right)=0$, to be fulfilled. The associated resonant term, in the sum (24), dominates the other bounded oscillating components. We can average out the oscillating part of $a$ to obtain the growth of the secular part $a_{S}$.

$$
\frac{d a_{S}}{d t}=\omega_{p}^{4} \frac{A^{3}}{64 M^{4}} J_{N}(N \varepsilon) .
$$

The density modulation can be excited in the high-frequency part of the ion acoustic wave dispersion or in the lowfrequency part. Consider the latter case, with a frequency far below the ion plasma frequency; for example, $\Omega_{i} \approx$ $\omega_{p i} \sqrt{T \mathrm{keV} / 511}$, and a dense plasma such that $\omega \approx \omega_{p}$. With these parameters, the $N \approx 1$ resonance can be achieved in the intensity range $A \approx 1$. For a weak modulation, we can use the expansion of the Bessel function in (23) to obtain

$$
a_{S}=\frac{\omega_{p}^{4} A^{3}}{128 M^{4}} \varepsilon \omega t
$$

Thus, the typical time needed to obtain third harmonic conversion of the pulse is of the order of $10^{2} / \varepsilon \omega(a \approx A \approx$ $\left.1, \omega \approx \omega_{p}\right)$.

To assess the impact of the variation of the various parameters on the resonant phase matching, three issues are to 
be addressed: 1) the amplitude stability, i.e., the impact of a small deviation $\delta \varepsilon$ of the amplitude of the modulation; 2) the frequency stability problem, i.e., the impact of a small detuning $\delta \Omega$; and 3 ) the effect of density inhomogeneities $\delta \omega_{p}$.

A variation of the amplitude of the modulation does not affect the resonance condition, but it modifies the time required to achieve the conversion. On the other hand, the modulation frequency and the background density must be controlled carefully. For a fixed $N$, the sine in (24) is a constant at exact resonance; if the resonance is slightly detuned by an amount $\delta \omega_{p}$ or $\delta \Omega$, then the sine term remains approximately constant during the time needed to perform the phase matched third harmonic conversion if

$$
|\delta \Omega|+\left|\delta \omega_{p}\right|<10^{-2} \varepsilon \omega .
$$

Although the saturation due to phase velocity mismatch is avoided, and linear growth recovered, the group velocity mismatch will ultimately limit the interaction time. This limitation is not severe, and it may be that a delay device can also achieve group velocity matching.

\section{CONCLUSION}

In quantum electronics, the most important issue of harmonic generation with bounded electrons is phase velocity matching: the same problem is also central to third harmonic generation with relativistic free electrons in plasma.

We show here that third harmonic generation can be achieved with an efficiency

$$
\frac{P_{3}}{P_{1}} \approx\left(\frac{3}{64}\right)^{2}\left(\frac{\omega_{p}}{\omega}\right)^{8} \frac{A^{4}}{M^{8}}\left[J_{N}(N \varepsilon) \omega t\right]^{2}
$$

in a density modulated plasma. The analytic techniques [7] introduced might also be employed to address the important issues of pump depletion, and the competition with the generation of the higher harmonics.

\section{ACKNOWLEDGMENT}

We gratefully acknowledge useful discussions with E. Valeo, P. Sprangle, J. Dawson, T. Johnston, and W. Mori.

\section{REFERENCES}

[1] P. Sprangle, E. Esarey, and A. Ting, "Nonlinear theory of intense laserplasma interaction,” Phys. Rev. Lett., vol. 64, pp. 2011-2014, 1990 $\{-\}\{-\}$, "Nonlinear interaction of intense laser pulses in plasma," Phys. Rev. A, vol. 41, pp. 4463-4469, 1990. A. Ting, E. Esarey, and P. Sprangle, "Nonlinear wake-field generation and relativistic focusing of intense laser pulses in plasmas," Phys. Fluids. B, vol. 2, pp. 1390-1394, 1990.

[2] M. Pessot, J. Squier, G. Mourou, and D. J. Harter, "Chirped-pulse amplification of 100 -fs pulses," Opt. Lett., vol. 14, pp. 797-800, 1987. M. Pessot, J. Squier, P. Bado, G. Mourou, and D. J. Harter, "Chirpedpulse amplification of 300 -fs pulses in an alexandrite regenerative amplifier," IEEE J. Quantum Electron., vol. OE-25, pp. 61-64, 1989.

[3] E. S. Sarachik and G. T. Schappert, "Classical theory of the scattering of intense laser radiation by free electrons," Phys. Rev. D, vol. 10, pp. 2738-2753, 1970. J. E. Gunn and J. P. Ostriker, "On the motion and radiation of charged particles in strong electromagnetic waves, motion in plane and spherical waves," Astro. J., vol. 165, pp. 523-541, 1971. R. E. Waltz and O. P. Manley, "Synchrotron-like radiation from intense laser beams in denses plasmas," Phys. Fluids, vol. 21, pp. 808-814, 1978.

[4] T. W. Kibble, "Frequency shift in high-intensity Compton scattering," Phys. Rev., vol. 138, pp. 740-753, 1965. J. H. Eberly and H. R. Reiss, "Electron self-energy in intense plane-wave field," Phys. Rev., vol. 145, pp. 1035-1040, 1966. T. W. Kibble, "Mutual refraction of electrons and photons," Phys. Rev., vol. 150, pp. 1060-1069, 1966.

[5] J. M. Rax and N. J. Fisch, "Third harmonic generation with ultra-high intensity laser pulses," Phys. Rev. Lett., vol. 69, pp. 772-775, 1992. P. Sprangle and E. Esarey, "Interaction of ultrahigh laser fields with beams and plasmas," Phys. Fluids B, vol. 4, pp. 2241-2244, 1992. E. Esarey et al., IEEE Trans. Plasma Sci., see this issue, pp. 95-104. W. Mori et al., IEEE Trans. Plasma Sci., see this issue, pp. 110-119.

[6] J. M. Dawson, "Nonlinear electron oscillation in a cold plasma," Phys. Rev., vol. 113, pp. 383-387, 1958. R. C. Davidson and P. P. Schram, "Nonlinear oscillations in a cold plasma," Nucl. Fusion, vol. 8, pp. 183-195, 1968.

[7] J. M. Rax and N. J. Fisch, "Nonlinear relativistic interaction of an ultrashort laser pulse with a cold plasma," Phys. Fluids B, vol. 4, pp. 1323-1331, 1992.

J. M. Rax, photograph and biography not available at the time of publication.

N. J. Fisch, photograph and biography not available at the time of publication. 